\title{
Changes in global gene expression of Vibrio parahaemolyticus induced by cold- and heat-stress
}

Sara Urmersbach ${ }^{1}$, Tommi Aho², Thomas Alter ${ }^{1}$, Syeda Sakira Hassan ${ }^{2,3}$, Reija Autio ${ }^{3,4}$ and Stephan Huehn ${ }^{*}$

\begin{abstract}
Background: Vibrio (V.) parahaemolyticus causes seafood-borne gastro-intestinal bacterial infections in humans worldwide. It is widely found in marine environments and is isolated frequently from seawater, estuarine waters, sediments and raw or insufficiently cooked seafood. Throughout the food chain, V. parahaemolyticus encounters different temperature conditions that might alter metabolism and pathogenicity of the bacterium. In this study, we performed gene expression profiling of $V$. parahaemolyticus RIMD 2210633 after exposure to $4,15,20,37$ and $42{ }^{\circ} \mathrm{C}$ to describe the cold and heat shock response.

Methods: Gene expression profiles of V. parahaemolyticus RIMD 2210633 after exposure to 4, 15, 20,37 and $42{ }^{\circ} \mathrm{C}$ were investigated via microarray. Gene expression values and RT-qPCR experiments were compared by plotting the $\log 2$ values. Moreover, volcano plots of microarray data were calculated to visualize the distribution of differentially expressed genes at individual temperatures and to assess hybridization qualities and comparability of data. Finally, enriched terms were searched in annotations as well as functional-related gene categories using the Database for Annotation, Visualization and Integrated Discovery.
\end{abstract}

Results: Analysis of $37^{\circ} \mathrm{C}$ normalised transcriptomics data resulted in differential expression of 19 genes at $20^{\circ} \mathrm{C}, 193$ genes at $4{ }^{\circ} \mathrm{C}, 625$ genes at $42^{\circ} \mathrm{C}$ and 638 genes at $15^{\circ} \mathrm{C}$. Thus, the largest number of significantly expressed genes was observed at 15 and $42{ }^{\circ} \mathrm{C}$ with 13.3 and $13 \%$, respectively. Genes of many functional categories were highly regulated even at lower temperatures. Virulence associated genes (tdh1, tdh2, toxR, toxS, vopC, T6SS-1, T6SS-2) remained mostly unaffected by heat or cold stress.

Conclusion: Along with folding and temperature shock depending systems, an overall temperature-dependent regulation of expression could be shown. Particularly the energy metabolism was affected by changed temperatures. Whole-genome gene expression studies of food related pathogens such as $V$. parahaemolyticus reveal how these pathogens react to stress impacts to predict its behaviour under conditions like storage and transport.

Keywords: Vibrio parahaemolyticus, Gene expression, Thermal shock

\section{Background}

Vibrio (V.) parahaemolyticus is one of the causes of seafood-borne gastro-intestinal infections in humans worldwide [1]. It is widely found in marine environments and is isolated frequently from seawater, estuarine waters, sediments and raw or insufficiently cooked seafood (e.g. shrimp or bivalve molluscs) [2-4]. Consumption of or contact to raw or undercooked seafood containing $V$.

\footnotetext{
* Correspondence: stephan.huehn@fu-berlin.de

'Institute of Food Hygiene, Freie Universität Berlin, Berlin, Germany

Full list of author information is available at the end of the article
}

parahaemolyticus in relevant numbers, might lead to human infections, mostly associated with gastroenteritis $[5,6]$.

Different studies investigated the behaviour of $V$. parahaemolyticus under environmental stresses on the phenotypic level (e.g. cold shock, heat shock, high salt concentrations or bile supplementation) [7-9]. Nonetheless, the general mechanism of adaptation and survival under these conditions are not elucidated yet.

Within its ecological habitat and food chain, V. parahaemolyticus encounters changing temperature conditions. These temperature shifts will result in metabolic changes. 
A cold shock resulting from a rapid downshift of the temperature, e.g. changing water temperatures or storage on ice, alters bacterial gene expression [10-13]. However the expression of $V$. parahaemolyticus resulting from cold shock is still poorly understood. The coldinduced gene expression profile of a clinical $V$. parahaemolyticus strain at $10{ }^{\circ} \mathrm{C}$ has been examined by Yang et al. [13] in a time course analysis. Significant differential expression of almost $13 \%$ of genes $(n=$ 619) investigated, was found.

Temperatures in the marine habitat of $V$. parahaemolyticus usually do not exceed $25^{\circ} \mathrm{C}$. In $V$. parahaemolyticus several stress proteins, e.g. heat shock protein (hsp) families such as Hsp60 (GroEL and GroES) and as Hsp70 (DnaJ, DnaK, GrpE) are produced in response to elevated temperatures $[14,15]$. In general those proteins are made in substantial amounts acting as chaperones, protecting cells from heat dependent denaturation [16-18]. In $V$. parahaemolyticus especially Hsp60 family proteins serve as general stress proteins and are found in several cell compartments and in substantial amounts [19].

In addition, changing temperature conditions can affect the pathogenicity of $V$. parahaemolyticus [19]. Chiang and Chou [20] demonstrated increased pathogenicity after heat shock response in $V$. parahaemolyticus as elevated toxin expression. Clinical strains alter expression of systems regulating virulence as well as systems indirectly related to host-pathogen attachment such as biofilm production and motility at $37{ }^{\circ} \mathrm{C}$ [21]. However, environmental strains did not show this behaviour or exhibit decreased expression of biofilm production or motility related genes at higher temperatures. Sublethal heat shock of $V$. parahaemolyticus resulted in elevated expression levels of the gene encoding the thermostable direct hemolysin (TDH), one of the two prominent toxins enhancing its pathogenicity [19].

The aim of this study was to investigate gene expression profiles of $V$. parahaemolyticus after exposure to 4 , 15, 20, 37 and $42{ }^{\circ} \mathrm{C}$. Moreover high regulation clusters e.g. toxins produced in response to temperature changes were to be identified.

\section{Results and discussion}

Understanding temperature-dependent changes in bacterial gene expression patterns is crucial when studying tenacity, invasion, and environmental related viability of bacterial species. Temperature-dependent expression changes as cues for tenacity and persistence within matrixes such as food or hosts and environment has led to genetic approaches defining temperature-induced genes of pathogens [22-24]. However, temperature-dependent induction of genes is an arbitrary parameter because appropriate temperatures for comparison to any other temperature must be assumed. In this study, we investigated temperature-dependent gene expression of $V$. parahaemolyticus in comparable growth phases under different temperatures.

\section{Validation of microarray results}

To confirm the results of microarray data analysis, a quantitative RT-qPCR was used. Six house-keeping genes were chosen to compare the data of the two techniques, whereof four were applied in the multilocus sequence typing (MLST) scheme of $V$. parahaemolyticus [25]. The house-keeping genes were encoded on both chromosomes, with one exception $(\operatorname{csp} \mathrm{A}): \operatorname{csp} \mathrm{A}, d t d \mathrm{~S}$, groES, pvsA, pyrC and tnaA. Four additional MLST genes used for normalization: $p v u \mathrm{~A}, d n a \mathrm{E}, r e c \mathrm{~A}$ and one locus of the 16S-23S intergenic spacer region. Gene expression values of microarray and RT-qPCR experiments were compared by plotting the $\log _{2}$ values of both experiments against each other. An overall positive correlation $\left(R^{2}=0.7008\right)$ between the two techniques could be shown (Fig. 1). The similarity of replicate samples at different temperatures was studied using hierarchical clustering with correlation as the distance measure (Fig. 2a). The samples at $42{ }^{\circ} \mathrm{C}$ form the clearest cluster. Samples of 20 and $37{ }^{\circ} \mathrm{C}$ cluster according to the temperature. Moreover, volcano plots of microarray data were calculated to visualize the distribution of differentially expressed genes at individual temperatures and to assess quality and comparability of hybridizations (Fig. 2b). Additionally, volcano plots enable the quick identification of expression changes within the gene sets by combination of statistical tests (adjusted $p$-value) and magnitude of changes.

\section{Gene expression at $4{ }^{\circ} \mathrm{C}, 15^{\circ} \mathrm{C}, 20^{\circ} \mathrm{C}$ and $42{ }^{\circ} \mathrm{C}$}

We compared gene expression patterns within a temperature range of 4 to $42{ }^{\circ} \mathrm{C}$. Additionally, Database for Annotation, Visualization and Integrated Discovery (DAVID) analyses were performed, highlighting regulation of genes connected in metabolic pathways (Additional file 1). Among all conditions, the strongest expression changes (regarding the number of differentially expressed genes and intensity of expression changes) were observed at $15{ }^{\circ} \mathrm{C}$ ( $13.3 \%$ of all genes) and $42{ }^{\circ} \mathrm{C}$ ( $13 \%$ of all genes). Since the highest number of genes with stable expression was found at $37{ }^{\circ} \mathrm{C}$, this temperature was chosen as reference. Genes with an adjusted $p$-value $\leq 0.05$ and an absolute logarithmic fold change $\leq \pm 1.5$ were considered significantly stable expressed. To demonstrate the temperature-associated differences in gene expression changes, temperature experiments were clustered via $K$-means-clustering (Fig. 3). The $K$-means-clustering arranges genes showing comparable expression under all temperatures investigated. Some genes showing clear up-regulation in both extreme conditions [Fig. 3 - cluster eight, 275 genes including sugar transport 


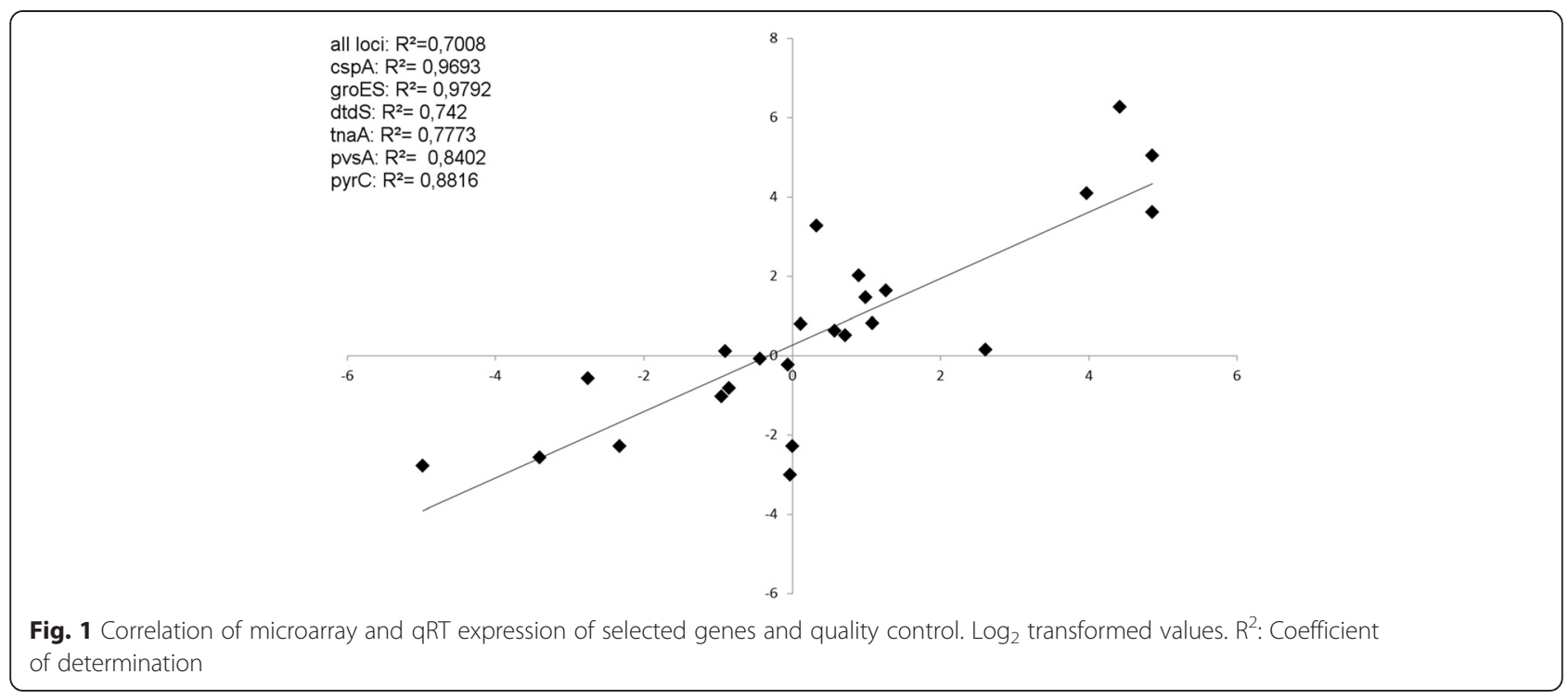

system permease (VP0908), tnaA (VPA0192) a tryptopanase, the putative translation elongation factor G, $p t f G$ (VPA0328), the putative phosphatase VPA0505, a putative membrane protein VPA1583] were found to be highly upregulated $\left(>2.0 \log _{2}\right)$. Genes down-regulated at 4 and $42{ }^{\circ} \mathrm{C}$ [Fig. 3 - cluster nine, 410 genes including the D-3phosphoglycerate dehydrogenase VP2593, eamA (VP2828) a pore forming protein and glyoxalase I (VP2166)] have been found in high numbers. In addition, there are genes down-regulated across all temperatures (Fig. 3 - cluster three, 154 genes including the putative proteases VP2447, VP2448 and an alcohol dehydrogenase VPA0870). Expression of genes sorted by chromosomes resulted in a higher rate of differentially expressed genes on the small chromosome (chromosome 2 ) at 15 and $42{ }^{\circ} \mathrm{C}$ (Table 1 ).

Our analysis identified differentially expressed genes under different temperature conditions. Compared to 37 at $4{ }^{\circ} \mathrm{C} 4 \%(n=193)$ the genes showed significant expression changes, whereas incubation at $20{ }^{\circ} \mathrm{C}$ resulted in a rate of approx. $0.4 \%(n=19)$. At $42{ }^{\circ} \mathrm{C}, 13 \%(n=$ $625)$ of differentially expressed genes were detected. The highest number of genes regulated, however, was found at $15{ }^{\circ} \mathrm{C}$ with $13.3 \%(n=638)$ differentially expressed genes. Incubation at 15 and $42{ }^{\circ} \mathrm{C}$ resulted in almost balanced expression patterns regarding the amount of upand down-regulated genes. At $4{ }^{\circ} \mathrm{C}, 78 \%(n=150)$ of the significantly differentially expressed genes showed down-regulation, whereas only $22 \%(n=43)$ showed up-regulation. Additional information can be found in Additional file 2.

\section{Expression of temperature shock response genes}

Some gene clusters showed up-regulation of expression under one temperature and down-regulation under another. Chaperone encoding $h s p 70$ family genes, such as
dnaK (VP0653), as well as the $h s p 60$ family groEL, groES (VPA0286, VPA0287) showed significant down-regulation of expression at 4,15 and $20{ }^{\circ} \mathrm{C}$. On the contrary, a strong up-regulation at $42{ }^{\circ} \mathrm{C}$ was observed (Additional file 2). Cold shock responding genes, such as cpsA (VPA12891291 and VP1889) as well as a cluster encoding genes classified as ascorbate and phosphotransferase (VPA0229231) showed significant up-regulation at 4,15 and $20^{\circ} \mathrm{C}$ whereas down-regulation occurred at $42{ }^{\circ} \mathrm{C}$. Yang et al. [13] investigated time dependent behaviour of a clinical $V$. parahaemolyticus strain at cold temperatures. Almost $13 \%$ of genes (619 genes) were differentially expressed at least at one of the three points in time investigated [13]. For metabolism related gene categories down-regulation was dominant over up-regulation due to the generally reduced cellular protein pool resulting from a sudden temperature downshift [11].

These findings are confirmed by our data. Moreover under cold temperatures, non-metabolic functions (cell envelope, transport and binding proteins, regulatory functions, cellular processes and mobile and extrachromosomal element functions) as well as genes with unknown or unassigned functions showed a more frequently up-regulation than genes related to cell structure and trans-membrane transporting functions (Additional file 1). The cold shock protein/regulator CspA (VPA1289) showed an over 30-fold enhanced transcription. Additionally, an antagonistic regulation of cold and heat shock genes was detected: heat shock genes encoding heat shock proteins $(h s p)$, ATP-dependent proteases and chaperons were mainly down-regulated after exposure to $10{ }^{\circ} \mathrm{C}$ [11-13]. Our results confirm the findings that metabolism related genes at low temperatures were mainly downregulated and genes without relation to metabolism or of unknown function were mainly up-regulated. Additionally, 



Fig. 2 Overview of microarray results. a The dendrogram represents the result of hierarchical clustering with euclidean distance measure. The first number in the sample label represents temperature, the second number is the replicate number at given temperature. $\mathbf{b}$ Volcano plot exemplarily shown for $15^{\circ} \mathrm{C}$ data. The $x$-axis represents the $\log _{2}$ of the fold change plotted against the - $\log _{10}$ of the adjusted $p$-value. Red points indicate the differentially expressed genes with at least 2.0 fold change and statistical significance adjusted $p<0.05$

antagonistic expression of cold and heat shock genes as well as a strong induction of cold shock proteins at low temperatures was observed for $V$. parahaemolyticus RIMD 2210633 (Additional file 2).

\section{Gene expression at $4{ }^{\circ} \mathrm{C}$ and $15{ }^{\circ} \mathrm{C}$}

At $4{ }^{\circ} \mathrm{C}$, only $4 \%$ of the genes were differentially expressed. Primarily transcription regulators as well as RNA metabolic process clusters were up-regulated, highlighting the impact of low temperatures $\left(4^{\circ} \mathrm{C}\right)$ on the overall gene expression (Fig. 4). Phadtare et al. [12] described concordant findings in E. coli. In our study, at $4{ }^{\circ} \mathrm{C}$ mainly genes encoding hypothetical proteins, e.g. VP1888, VP2889, VP3030 and VPA1291 were up-regulated.
Additionally, genes of the energy metabolism (VP1381, VP1533, VP2005, VP2666, VP2987, VPA0092) reacted to $4{ }^{\circ} \mathrm{C}$ by up-regulation. Especially VP1533, encoding a putative ATPase, is of great importance for energy production using glucose [26]. In particular cold shock proteins were highly expressed (cspA VP1889, $4.05 \log _{2}$ fold change). These findings, originally described by Yang et al. [13], were confirmed by our data. However, cold temperatures bias gene expression results due to lower activities of e.g. enzymes [27].

The global regulator sigma factor 38, rpoS (VP2553) and the osmoregulator $0 m p \mathrm{R}$ (VP0154) were up-regulated (3.5 and $4.1 \times$ ). Sigma factor 38 is one of the most crucial sigma factors under e.g. extreme temperatures [28]. No 

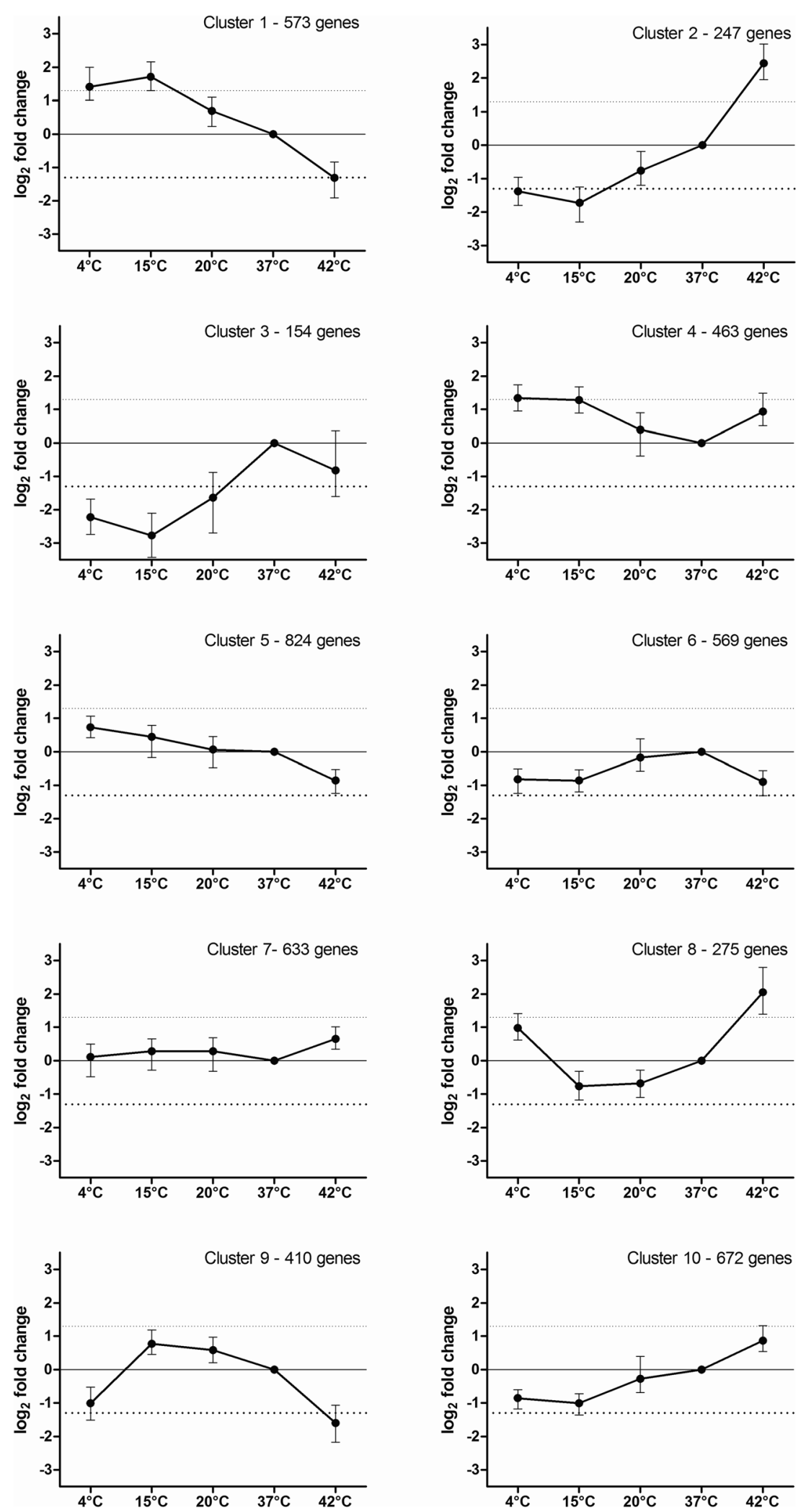

Fig. 3 Clustering of genes with similar expression patterns. Ten clusters of similar expressed genes at 4, 15, 20 and $42^{\circ} \mathrm{C}$ normalized to $37^{\circ} \mathrm{C}$ are shown. The incubation temperatures ( $x$-axis) where plotted against the $x$-fold gene expression ( $y$-axis) of genes sorted in the particular box. Clustering was performed using $K$-means (genesis) 
Table 1 Differentially expressed genes according to encoding chromosome

\begin{tabular}{lrrrr}
\hline Incubation temperature & \multicolumn{1}{c}{ Chr1-up } & \multicolumn{1}{c}{ Chr1-down } & \multicolumn{1}{c}{ Chr2-up } & $35(2.00 \%)$ \\
\hline $4{ }^{\circ} \mathrm{C}$ & $116(3.77 \%)$ & $26(0.85 \%)$ & $147(8.41 \%)$ & $16(0.92 \%)$ \\
$15{ }^{\circ} \mathrm{C}$ & $214(6.96 \%)$ & $171(5.56 \%)$ & $2(0.11 \%)$ & $107(6.12 \%)$ \\
$20^{\circ} \mathrm{C}$ & $3(0.10 \%)$ & $9(0.29 \%)$ & $187(10.70 \%)$ & $5(0.29 \%)$ \\
$42{ }^{\circ} \mathrm{C}$ & $146(4.75 \%)$ & $204(6.64 \%)$ & $88(5.04 \%)$ \\
\hline
\end{tabular}

The numbers of differentially expressed genes are given in total as well as in proportion to the number of genes present on the microarray in brackets. Chr1: genes encoded on chromosome 1 encoding 3080 genes of which 3073 genes were represented on the array. Chr2: genes encoded on chromosome 2 encoding 1752 genes of which 1747 genes were represented on the array. Down: down-regulated genes; up: up-regulated genes

other sigma factors were up-regulated. Additionally, the tRNA methyltransferase spoU (VP0158) described by Persson et al. [29] was up-regulated $(4.1 \times)$ as well. Persson et al. [29] were not able to detect differences in growth rates of the E. coli wild-type and a spoU mutant. However, growth temperatures were between 37 and $42{ }^{\circ} \mathrm{C}$ in that study. Maybe a particular part of tRNA activation can be triggered by low temperatures. Since none of the known genes related to DNA damage VP2034 (imuA), VP2035 (imuB), VP2036 (dnaE2), VP2550 (recA) and VP2945 (lexA) were up-regulated, cold induced DNA damaging, triggering the SOS response, appears to be absent. At $4{ }^{\circ} \mathrm{C}$ 11 DAVID-gene categories were identified in which a statistically significant number of genes $(n=186, p$-value $<0.05)$ was differentially regulated. Nine of these categories were related to transcription, DNA-binding and regulation of RNA metabolism. The two other categories were related to ABC-transporters or transmembrane domains. The expression of genes organized into functional categories at $4{ }^{\circ} \mathrm{C}$ is shown in Fig. 5. The top five up- and down-regulated genes at $4{ }^{\circ} \mathrm{C}$ are shown in Table 2.

At $15{ }^{\circ} \mathrm{C}$ a total of 638 genes were differentially expressed (Additional file 2). In addition to rpoS, omp R and spoU, transcriptional regulators VP0034, VP0059, VP0713, VP1391, VP1676, VP1765, VPA0214, VPA1219 and VPA1289 were up-regulated, along with DNA repair VP2943, VPA1393, DNA polymerase III (VP2036), DNA integrase (VP1071). Thus, partially DNA repair has been up-regulated along with protein and peptide secretion and trafficking (VPA1208, VPA1209, VPA1443, VPA 1445). However, no genes related to SOS repair, such as recA (VP2550) and lexA (VP2945), or global stress regulators such as $h f q$ were up-regulated. These regulators seem of minor concern under these circumstances described. However, strong up-regulation was found for putative regulators such as VP1391 (5x) and VPA1219 (8x).

Especially energy metabolism was down-regulated; out of 75 differentially expressed genes of this category 65

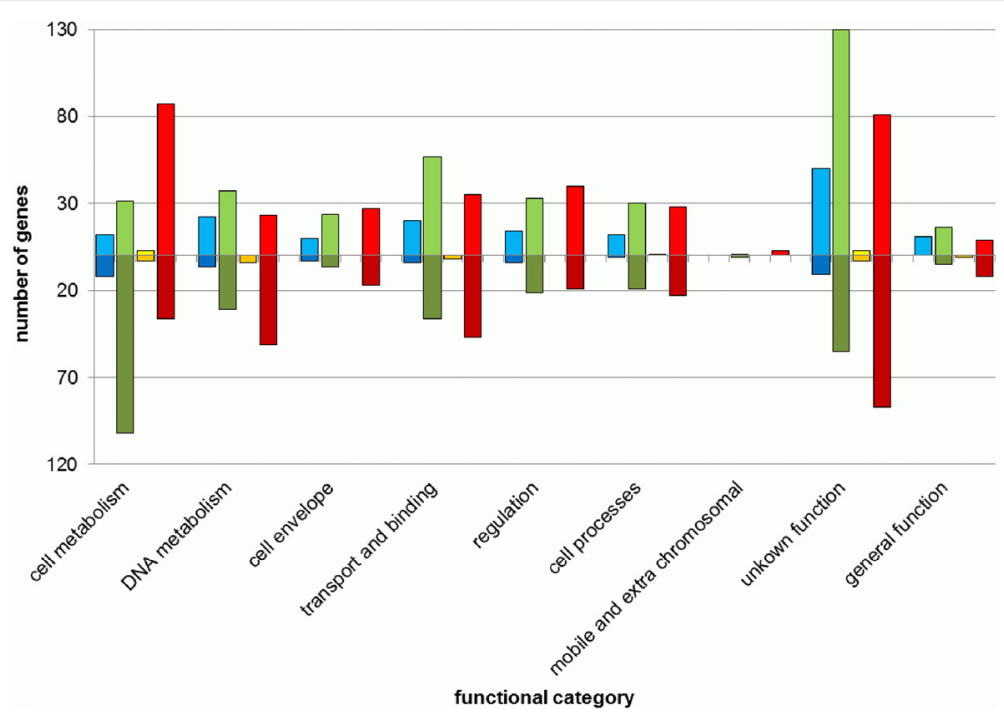

Fig. 4 Functional annotation of differentially expressed genes. The amount of genes was plotted according to their function and incubation temperature. Only significantly expressed genes are shown with at least $1.5 \log _{2}$ fold change of expression rate. Normalized incubation temperatures are shown in blue, $4^{\circ} \mathrm{C}$; green, $15^{\circ} \mathrm{C}$; yellow, $20^{\circ} \mathrm{C}$ and red, $42^{\circ} \mathrm{C}$. Dark shading of colour indicates down regulation at the corresponding temperature. All differentially expressed genes with a $\log _{2}$ fold change $>1.5$ and adjusted $p$-value $<0.05$ in each condition are supplied in Additional file 2 


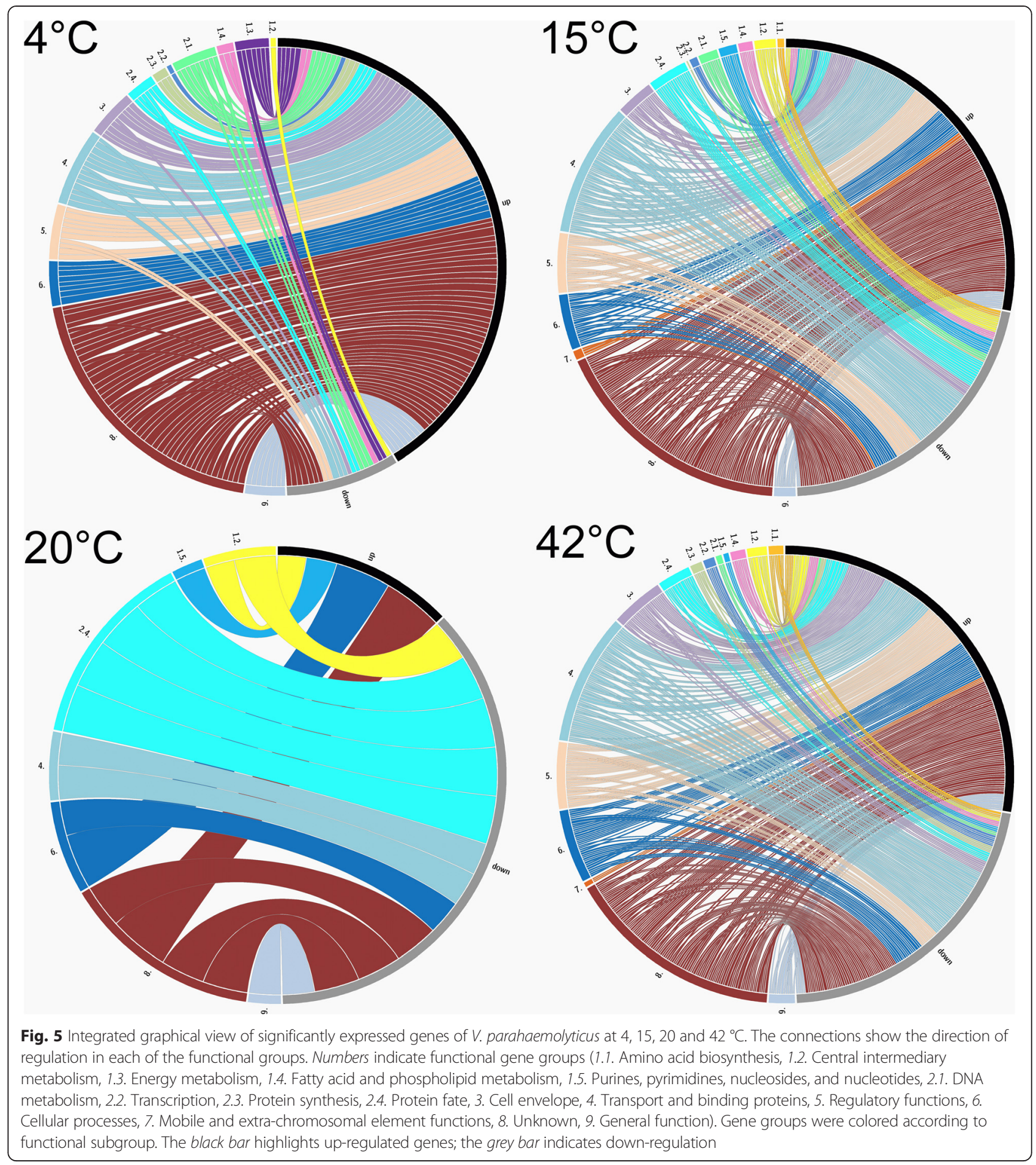

genes $(87 \%)$ were repressed (Fig. 4). Genes related to the pentose phosphate pathway, glycolysis and the citric acid cycle were down regulated. We suggest, that production of central energy molecules such as ATP, NADPH and NADH was decreased because of down-regulated expression of corresponding genes. The vast majority of genes showing highest up-regulation, however, were of unknown function (Additional file 2). The putative virulence-associated protein $\mathrm{VacB}$ showed highest upregulation $(128 \times)$, which has been described to react to environmental signals in Haemophilus influenza [30].

Gene expression of functional categories is shown in Fig. 5. In contrast to $4{ }^{\circ} \mathrm{C}$ incubation, genes of the amino acid category and de novo DNA synthesis were induced 
Table 2 Top 5 up- and down-regulated genes at $4^{\circ} \mathrm{C}$

\begin{tabular}{|c|c|c|c|c|}
\hline \multirow[b]{2}{*}{ Gene ID } & \multirow[b]{2}{*}{ Gene } & \multirow[t]{2}{*}{ Protein } & \multirow{2}{*}{$\begin{array}{r}\log _{2} \mathrm{fc} \\
4^{\circ} \mathrm{C} \\
\end{array}$} & \multirow[t]{2}{*}{ adj.p-value } \\
\hline & & & & \\
\hline VP1717 & & ABC-type multidrug & -3.66 & 0.024 \\
\hline VP2024 & & hypothetical protein & -3.27 & 0.017 \\
\hline VP0502 & & hypothetical protein & -2.83 & 0.035 \\
\hline VP2264 & & hypothetical protein & -2.80 & 0.035 \\
\hline VP2445 & & put. lipid carrier protein & -2.78 & 0.018 \\
\hline VP1889 & $\operatorname{cspA}$ & cold shock transcr. reg. & 4.06 & 0.022 \\
\hline VP2889 & & hypothetical protein & 4.15 & 0.019 \\
\hline VP3030 & & hypothetical protein & 4.46 & 0.009 \\
\hline VPA1291 & & hypothetical protein & 4.74 & 0.029 \\
\hline VP1888 & & hypothetical protein & 4.86 & 0.013 \\
\hline
\end{tabular}

Coloured boxes highlight at least 1.5 fold differential expression in either direction: red up-regulated, green down-regulated, fc (fold change) is given log $\mathrm{g}_{2}$ transformed; transcr. reg. transcriptional regulator, put. putative

at 15 and $42{ }^{\circ} \mathrm{C}$. In total 32 DAVID-gene categories with 475 differentially expressed genes were identified at $15^{\circ} \mathrm{C}$. Ten categories were associated with transport and transporters. Two major groups formed the most important clusters: integral and intrinsic components of the membrane with 69 (11.6 \%) genes each. Additionally, nine metabolism related categories were identified.

At $15{ }^{\circ} \mathrm{C} 32$ DAVID-gene categories with 475 genes showed differential regulation. Many of them were connected with membrane maintenance or metabolism. In E. coli it could be shown that at $12{ }^{\circ} \mathrm{C}$ the membrane composition remains unchanged but enzyme activations are effected [31]. The top five up- and down-regulated genes at $15^{\circ} \mathrm{C}$ are shown in Table 3 .

\section{Gene expression at $20^{\circ} \mathrm{C}$}

At $20^{\circ} \mathrm{C}$, no differential expression of metabolic pathways was detectable. Thus, a range of temperature between 15 and $20{ }^{\circ} \mathrm{C}$ seems to describe the (lower) physiological border of the normal condition for the strain investigated. A total of 19 genes was differentially expressed. Gene expression is shown in Fig. 5. At $20{ }^{\circ} \mathrm{C}$ solely genes related to categories associated with the degradation of peptides and proteins were identified: 'peptidase' (15.8\%), 'protease', 'peptidase activity' and 'proteolysis' (21.1\%), respectively. The top five up- and down-regulated genes at $20{ }^{\circ} \mathrm{C}$ are shown in Table 4.

\section{Gene expression at $42{ }^{\circ} \mathrm{C}$}

At $42{ }^{\circ} \mathrm{C}$ transport and metabolism of carbohydratesrelated genes were up-regulated (Additional file 2). Again, a range of temperature between 42 and $37{ }^{\circ} \mathrm{C}$ seems to describe the (upper) physiological border for the clinical strain investigated. Interestingly, more genes located on the small chromosome were differentially expressed during incubation at all temperatures. Especially, at $42{ }^{\circ} \mathrm{C}$ almost twice as many small chromosome genes were differentially expressed. The higher intensity of expression

Table 3 Top 5 up- and down-regulated genes at $15^{\circ} \mathrm{C}$

\begin{tabular}{lrlrr}
\hline Gene ID & Gene & Protein & $\begin{array}{r}\log _{2} \mathbf{f c} \\
\mathbf{1 5}^{\circ} \mathbf{C}\end{array}$ & adj. p-value \\
\hline VP2362 & ompK & OmpK precursor & -6.10 & 0.002 \\
VPA0230 & & put. PTS component II B & -6.05 & 0.007 \\
VPA1084 & rbsB & D-ribose transporter & -5.48 & 0.049 \\
VPA0231 & & Phosphotransferase & -5.42 & 0.004 \\
VPA0229 & ulaA & ascorbate-specific PTS & -5.25 & 0.005 \\
VP1889 & cspA & cold shock transcr. reg. & 4.41 & 0.007 \\
VPA1413 & & hypothetical protein & 4.44 & 0.004 \\
VPA1289 & cspA & cold shock transcr. reg. & 4.86 & 0.014 \\
VP1888 & & hypothetical protein & 6.55 & 0.004 \\
VP1890 & \multirow{2}{*}{ vacB } & VacB/RNase R & 7.01 & 0.006 \\
\hline
\end{tabular}


Table 4 Top 5 up- and down-regulated genes at $20^{\circ} \mathrm{C}$

\begin{tabular}{lrlrl}
\hline Gene ID & Gene & Protein & $\begin{array}{r}\log _{2} \mathbf{f c} \\
\mathbf{2 0}{ }^{\circ} \mathbf{C}\end{array}$ & $\begin{array}{r}\text { adj. } \mathbf{p}- \\
\text { value }\end{array}$ \\
\hline VPA0611 & ackA & acetate kinase & -4.60 & 0.031 \\
VPA1193 & pepT & peptidase T & -3.92 & 0.036 \\
VP2448 & & put. protease & -3.85 & 0.010 \\
VP2447 & & put. protease & -3.81 & 0.010 \\
VP0990 & & hypothetical protein & -3.56 & 0.010 \\
VPA0071 & & put. alcohol dehydrogenase & 2.08 & 0.042 \\
VP1721 & & aminotransferase & 2.25 & 0.042 \\
VPA1159 & guaC & guanosine oxidoreductase & 2.39 & 0.018 \\
VP1889 & cspA & cold shock transcr. reg. & 4.38 & 0.042 \\
VP1888 & & hypothetical protein & 4.75 & 0.031 \\
\hline
\end{tabular}

Coloured boxes highlight at least 1.5 fold differential expression in either direction: red up-regulated, green down-regulated, fc (fold change) is given log ${ }_{2}$ transformed; transcr. reg. transcriptional regulator, put. putative

changes in genes located on the small chromosome compared to genes located on the large chromosome can be explained by the higher number of genes related to transcriptional regulation and transport of various substances being located on the small chromosome [32]. Thus, most genes related to environmental stress response are encoded on the small chromosome.

Primarily, genes classified as 'cell metabolism' along with the genes classified as 'unknown', reacted to the temperature upshift. Altogether, the expression of 625 genes was differentially expressed at $42{ }^{\circ} \mathrm{C}$. Expression of categorized genes is shown in Fig. 5. Out of the 87 'cell metabolism' genes, $55 \%(n=48)$ were classified as 'energy metabolism' related genes.

A wide spectrum of genes was affected. For example, genes associated with amino acid and amine synthesis (pyruvate family) were induced, whereas genes related to histidine (VP1137), serine (VP1324, VP1629, VP2593) and aromatic amino acid (VP2744, VP3065) families were down-regulated (Additional file 1). Out of 55 energy metabolism related genes only six were downregulated in expression. Particularly, genes of electron transfer (VP1161, VPA0643, VPA0949, VPA1428), biosynthesis of polyamines (VPA0169, VPA0170, VPA1635) and degradation of fatty acids as well as fermentation (VP1647, VP2543, VPA0478, VPA0502, VPA1416) were up-regulated at $42{ }^{\circ} \mathrm{C}$. Moreover especially sugar metabolism (VP1303, VP2397, VP2398, VP2400, VPA1674, VPA1675, VPA1700, VPA1706) was affected (Additional file 1). Genes involved in arabinose (VPA 1671-1678), mannose and glucoronate (VPA1702-1709) metabolism and transport were up-regulated. Additionally heat protection protein encoding genes such as groEL, groES were induced. Reactions of heat shock proteins such as GroEL/GroES, are in concordance with data described by Wong et al. [19].
At $42{ }^{\circ} \mathrm{C}, 38$ DAVID-gene categories with a total of 423 differentially expressed genes were identified (Additional file 1). Amongst others, nine categories were related to cell-motion (flagella), eight categories to metabolic processes and six categories to RNA, DNA and transcription. Additionally, three categories were associated with homeostasis (ion, cation, chemical) and two categories with iron-siderophores and transport of siderophores. A distinct cluster on the second chromosome encoding the genes VPA0915-1042 ('cellular processes': $n=23$, 'energy metabolism': $n=18$, 'transport and binding': $n=14$, 'regulatory functions': $n=14$ and 'unknown': $n=36$ ) showed upregulation at $42{ }^{\circ} \mathrm{C}$. The top five up- and down-regulated genes at $42{ }^{\circ} \mathrm{C}$ are shown in Table 5 .

However, no prior studies about genome wide gene expression responses exist for the temperatures investigated in this study.

\section{Temperature dependent expression of virulence genes}

Virulence genes in total showed no significant expression changes under different temperatures (Additional file 2). The expression of $t d h$ was not significantly influenced by temperature changes, even though slight activation (2.1 $\log _{2}$ fold change) was observed at $15^{\circ} \mathrm{C}$. A putative haemolysin encoding gene (VP3048), was up-regulated at 4 and $15{ }^{\circ} \mathrm{C}$. This effect was described by Yang et al. [13], reporting an induction of this putative haemolysin after cold shock. The most prominent haemolysin $t d h$, however, was not significantly up-regulated (Additional file 2). The associated regulator opa $\mathrm{R}$ which recently has been shown to repress expression of T6SS in $V$. parahaemolyticus is down-regulated at $42{ }^{\circ} \mathrm{C}$ [33]. We found that, genes located within the virulence pathogenicity island 7 (VPa-7) encoded on the small chromosome, VPA1312-1396 showed no reaction to thermal stimulations (Additional file 2). However, since the energy metabolism was affected 
Table 5 Top 5 up- and down-regulated genes at $42^{\circ} \mathrm{C}$

\begin{tabular}{lllrr}
\hline Gene ID & Gene & Protein & $\begin{array}{r}\log _{2} \mathbf{f c} \\
\mathbf{4 2}{ }^{\circ} \mathbf{C}\end{array}$ & adj. p-value \\
\hline VP0712 & & hypothetical protein & -5.64 & $8.16546 \mathrm{E}-07$ \\
VPA1424 & & PTS system & -5.42 & 0.016 \\
VP0053 & & hypothetical protein & -5.10 & 0.011 \\
VPA1289 & cspA & cold shock transcr. reg. & -4.98 & 0.022 \\
VPA1425 & manA & mannose-6-p isomerase & -4.89 & 0.010 \\
VP0084 & & hypothetical protein & 5.57 & 0.026 \\
VP2479 & & peptide ABC transporter & 5.57 & 0.002 \\
VPA0505 & & put. hydrolase & 6.13 & 0.010 \\
VPA0286 & groES & co-chaperonin GroES & 6.24 & 0.005 \\
VPA0287 & groEL & chaperonin GroEL & 6.66 & 0.004 \\
\hline
\end{tabular}

Coloured boxes highlight at least 1.5 fold differential expression in either direction: red up-regulated, green down-regulated, fc (fold change) is given log ${ }_{2}$ transformed; transcr. reg. transcriptional regulator, put. putative

especially and mostly at cold temperatures, reduced classical virulence or changed expression rates were to be expected [34].

Virulence associated genes in general $(t d h 1, t d h 2$, tox $\mathrm{R}$, toxS, vopC, T6SS-1: VP1386-1420), remained unaffected by heat or cold stress (Additional file 2). The T3SS-1 was found down-regulated at $15{ }^{\circ} \mathrm{C}$ for nos A (VP1697) and up-regulated for the putative chaperone VP1687 at $42{ }^{\circ} \mathrm{C}$. However, the T6SS-1 located on chromosome 1 showed up-regulation at $42{ }^{\circ} \mathrm{C}$. This was to be expected since the T6SS-1 system reacts to warm climate in $V$. parahaemolyticus as described by Salomon et al. [35]. The cold shock gene $\operatorname{csp} \mathrm{A}$ was downregulated, whereas heat shock genes encoding chaperones and protection via sugar metabolites were induced [13].

\section{Conclusions}

Based on our data, the optimal temperature range of the clinical $V$. parahaemolyticus strain investigated is between 20 and $37{ }^{\circ} \mathrm{C}$, since most of the genes were transcribed at a rather constant level.

Finally, it could be shown that the classical pathogenicity markers, T3SSs as well as T6SSs were not upregulated in response to thermal changes. However, large proportions $(\sim 30 \%)$ of the differentially expressed genes are of unknown function. Summarized, this study successfully demonstrated that genome-wide gene expression changes in $V$. parahaemolyticus occur at 4, 15, 20, and $42{ }^{\circ} \mathrm{C}$.

\section{Methods}

\section{Bacterial strains}

V. parahaemolyticus RIMD2210633 was isolated from a patient suffering from diarrhoea in Japan in 1996 [32]. This strain harbours the $t d h$ gene, lacks the trh gene and belongs to serotype O3:K6 [36]. This serotype has been detected in clinical as well as in environmental marine samples [37]. The strain has been sequenced by Makino et al. [32].

Prior use, the strain was stored in cryovials at $-80{ }^{\circ} \mathrm{C}$ (Cryobank; Mast Diagnostica, Bootle, England). For initial growth, cells were grown using a rotary shaker (Unimax 1010 and Incubator 1000; Heidolph, Schwabach, Germany) in alkaline peptone water (APW; $0.3 \%$ Yeast-Extract, $1 \%$ Peptone, $2 \% \mathrm{NaCl} ; \mathrm{pH} 8.6)$ at $37{ }^{\circ} \mathrm{C}$ overnight. A $2 \mathrm{ml}$ aliquot of the resulting culture was diluted to a total volume of $25 \mathrm{ml}$ using APW and grown to an $\mathrm{A}_{600} \mathrm{~nm}$ of 0.6. Cultures were grown at $37{ }^{\circ} \mathrm{C}$ for $3.5 \mathrm{~h}$ in order to generate exponential phase cultures. After appropriate dilutions the $\mathrm{A}_{600}$ was analysed again and aliquots consisting of $10^{8}$ to $10^{9} \mathrm{~V}$. parahaemolyticus cells were transferred to $15 \mathrm{ml}$ Falcon tubes, placed in a thermal mixer (Thermomixer comfort; Eppendorf, Hamburg, Germany) and incubated at different temperatures $\left(42,37\right.$ and $20{ }^{\circ} \mathrm{C}$ ) for $30 \mathrm{~min}$. For stressing the cells at 4 and $15^{\circ} \mathrm{C}$ the entire incubation unit was placed in a conditioning cabinet (Rubarth Apparate, Laatzen, Germany) and bacteria were incubated at these temperatures for $30 \mathrm{~min}$.

\section{RNA preparation and reverse transcription for qPCR investigation}

The cultures were centrifuged $(2 \mathrm{~min}, 8000 \times \mathrm{g})$ and the supernatant was discarded. The pellet was immediately resuspended in $1.5 \mathrm{ml}$ RNAprotect Bacteria Reagent (Qiagen, Hilden, Germany) to minimize RNA degradation. Total RNA was isolated using the peqGold Bacterial RNA Kit (Peqlab, Erlangen, Germany). The obtained RNA was eluted into $43 \mu \mathrm{l}$ of DEPC-treated, DNaseand RNase-free water (Carl Roth, Karlsruhe, Germany). Samples were then treated with DNase I along with Ribolock, an RNase-A, $-\mathrm{B}$ and -C inhibitor (Fermentas, Vilnius, Lithuania). RNA quantity was measured by spectrophotometry. RNA quality of each sample was 
monitored via gel electrophoresis. Additionally, the RNA quality was assessed using the Agilent RNA 6000 Nano Kit on a 2100 Bioanalyzer (Agilent, Santa Clara, US).

Fluorescence-labeled cRNA generation for the microarray Prior to labelling, the RNA was initially transcribed in cDNA. Briefly, $200 \mathrm{ng}$ of RNA were linear amplified using the full spectrum MultiStart primer (Biocat, Heidelberg, Germany) and Moloney murine leukemia virus reverse transcriptase (Agilent). The amplification was performed at $40{ }^{\circ} \mathrm{C}$ for $2 \mathrm{~h}$ followed by $65{ }^{\circ} \mathrm{C}$ for $15 \mathrm{~min}$ and stored at $4{ }^{\circ} \mathrm{C}$. The amplified CDNA, the full spectrum MultiStart primer and T7 RNA polymerase were used along with Cyanine 3-CTP (Agilent) generating labelled cRNA. Labeling was performed using the Quick Amp Labeling Kit (Agilent). The labeled cRNA was purified using the Qiagen RNeasy Mini Kit (Qiagen). A $3 \mu \mathrm{l}$ aliquot was used for quality control. Experiments were performed using Agilent custom $8 \times 15 \mathrm{k}$ arrays (Agilent). The microarray field covers $99.75 \%$ of all $V$. parahaemolyticus genes. In total, 3073 out of 3080 genes encoded on chromosome 1 and 1747 out of 1752 genes located on chromosome 2, are included. Each gene is represented by 1 to 10 probes (mean 3.15 probes per gene). Each probe consists of a 60mer located preferentially at the 3' terminus of the corresponding gene. The probe design was performed with the eArray Software a web-based Agilent application basing on the genome sequence of $V$. parahaemolyticus RIMD 2210633 (http://www.ncbi.nlm.nih.gov/genome/691?genome_assembly_id=167995). The cRNA samples were then hybridized to an individual microarray field.

\section{Microarray hybridization and post hybridisation washing}

For hybridizations on the microarray, three replicates of independently grown bacterial cultures were prepared for each temperature condition, for $37{ }^{\circ} \mathrm{C}$ four replicates were used. Accordingly, three individually labeled cRNA sets were prepared for each temperature other than $37{ }^{\circ} \mathrm{C}$. Finally, $600 \mathrm{ng}$ of the labeled and linear amplified cRNA was fragmented, added to $25 \mu \mathrm{l}$ hybridization buffer mix of which a $20 \mu \mathrm{l}$ aliquot (480 ng) was loaded on a microarray in a hybridization chamber (Biometra, Goettingen, Germany). The onechannel hybridization was performed at $65{ }^{\circ} \mathrm{C}$ for $17 \mathrm{~h}$ and $10 \mathrm{rpm}$.

Washing of the slides was performed using preheated washing buffer (Gene expression wash buffer kit, Agilent). First the chamber was rinsed with washing buffer. Then the slides were washed once followed by a second washing step using washing buffer containing $0.01 \%$ Triton X-102 (Agilent). The slides were dried using acetonitrile.

Table 6 qRT-PCR primers

\begin{tabular}{|c|c|c|c|c|}
\hline Gene & ID & Sequence $5^{\prime}$ to $3^{\prime}$ & Size [bp] & Reference \\
\hline \multirow[t]{2}{*}{$1623 S$} & bp 134385 & GCTGACAAAACAACAATTTATTGTT & 170 & {$[45]$} \\
\hline & to $135166^{\mathrm{a}}$ & GGAGTTTCGAGTTGATGAAC & & \\
\hline \multirow[t]{2}{*}{ groES } & VP2852 & TATTCAACGATCGCCATGAT & 108 & This study \\
\hline & & TGGTGACACCGTTATCTTCG & & \\
\hline \multirow[t]{2}{*}{$\operatorname{csp} \mathrm{A}$} & VPA1289 & TATCGTTGCTGACGGTTTCA & 90 & This study \\
\hline & & TCAGTCGCTTGAGGACCTIT & & \\
\hline \multirow[t]{2}{*}{ pvsA } & VPA1658 & GGACCTCCACGTCGTTCTTA & 112 & This study \\
\hline & & GGGATTGAAGACATCGCACT & & \\
\hline \multirow[t]{2}{*}{ pvuA } & VPA1656 & GCTGTCGATGCTTGATCGTA & 107 & This study \\
\hline & & GTGGAATCGGTTTGGTCACT & & \\
\hline \multirow[t]{2}{*}{ recA } & VP2550 & GAAACCATTTCAACGGGTTC & 139 & {$[25]$} \\
\hline & & GTGCAGCAGCGATAAGCTC & & This study \\
\hline \multirow[t]{2}{*}{$d n a E$} & VP2303 & GATTACCGCTTTCGCCG & 140 & {$[25]$} \\
\hline & & GTGTATCCATGCCCGATTTC & & This study \\
\hline \multirow[t]{2}{*}{$d t d S$} & VPA1508 & TGGCCATAACGACATTCTGA & 124 & {$[25]$} \\
\hline & & TTCGTGACCGACAACCATAG & & This study \\
\hline \multirow[t]{2}{*}{ pyrC } & VPA0408 & AGCAACCGGTAAAATTGTCG & 142 & [25] \\
\hline & & TCCATGAACCAAAAGCAACA & & This study \\
\hline \multirow[t]{2}{*}{ thaA } & VPA0192 & TGTACGAAATTGCCACCAAA & 103 & {$[25]$} \\
\hline & & TCAGCGTAACCTTCTTCACG & & This study \\
\hline
\end{tabular}

a 16S-23S intergenic spacer region encoded on chromosome 2 


\section{Data handling and microarray analysis}

Scanning was carried out using the Agilent G2565CA scanner with a resolution of $5 \mu \mathrm{m}$. After scanning, tiff-files were analysed and raw data was extracted using Feature Extraction Software (Agilent). Data processing was performed using Bioconductor V 2.12 package of the software R. At first, background corrected spot intensities (signal gProcessedSignal in the Agilent protocol GE1_107_Sep09) were retrieved and bad quality spots were removed using the outlier detection flags of the Agilent protocol. Further, the signal values were normalized using quantile normalization and $\log _{2}$ transformed [38]. Linear modelling and empirical Bayes methods, implemented in the $R$ package Limma [39], were used to detect the differentially expressed genes between two groups, in this case, the control and treatment sample groups. Raw $p$-values were adjusted using the Benjamini and Hochberg multiple adjustment method [40]. Genes with an adjusted $p$-value $\leq 0.05$ and an absolute logarithmic fold change $\geq 1.5$ were considered significantly differentially induced, while genes with an absolute logarithmic fold change $\leq-1.5$ were considered repressed. Annotation of genes was performed according to Yang et al. [13] and updated using two new gene entries at NCBI (http://www.ncbi.nlm.nih.gov/ gene), KEGG (http://www.genome.jp/kegg/) and Gene Ontology (http://www.geneontology.org/).

Finally, enriched terms were searched in annotations as well as functional-related gene categories using the Database for Annotation, Visualization and Integrated Discovery (DAVID V 6.7, Fisher exact test) [41, 42]. The gene lists generated via DAVID enable to highlight gene sets which show a higher proportion of differentially expressed genes compared to other categories. This eases identification of pertinent biological processes to the according temperature. The identified categories are presented in the Additional file 1. K-means clustering of genes with similar gene expression was performed using Genesis V 1.7.6 [43]. Heat maps were generated using BioNumerics V 6.01 (Applied Math, St. Martens-Latem, Belgium). Volcano plots were generated via GraphPad V 5.04, (GraphPad, San Diego, US). Integrated graphical views were generated using Circos plot [44]. The transcriptomics data were supplied as experiment GSE60815 at Gene Expression Omnibus according to MIAME regulations. All differentially expressed genes with a $\log _{2}$ fold change $>1.5$ and adjusted $p$-value $<0.05$ of each condition are supplied in Additional file 2.

\section{qRT-PCR}

For generating cDNA, a $1 \mu \mathrm{g}$ RNA aliquot was used and reversely transcribed by the RevertAid Premium First
Strand cDNA Synthesis Kit and random hexamer primers according to the manufacturer's instructions (Fermentas). Additionally, $1 \mu \mathrm{g}$ of total RNA was used as RT- negative control following the same protocol with additional reaction buffer instead of the enzyme mix. Resulting cDNAs as well as RT-negative controls were diluted 1:50 in DNase- and RNase-free water. $1 \mu \mathrm{l}$ of each sample was used for qRT-PCR.

Specific oligonucleotide primer pairs were used for PCR (Table 6). New primers or new primer pairs were designed with Primer3 software (http://frodo.wi.mit.edu/) and synthesized (Metabion, Martinsried, Germany). The amounts of cDNA of all genes were determined by qRT-PCR assays in $12.5 \mu \mathrm{l}$ reaction volume. Conditions for the reactions were: $6.25 \mu \mathrm{l}$ of $2 \times$ SsoFast Eva Green Supermix (BioRad, Hercules, US), $0.5 \mu \mathrm{M}$ of each primer, $1 \mu \mathrm{l}$ of cDNA; $1 \times 95{ }^{\circ} \mathrm{C}$ for $3 \mathrm{~min}, 45 \times 95{ }^{\circ} \mathrm{C}$ for $10 \mathrm{~s}$ and $57{ }^{\circ} \mathrm{C}$ for $15 \mathrm{~s}$ in a BioRad C1000 cycler with an CFX96 optical head. Validation of specific products was done via melting curve analysis, consisting of an initial heating at $95{ }^{\circ} \mathrm{C}$ for $10 \mathrm{~s}$, followed by a stepwise temperature increase from 68 to $88{ }^{\circ} \mathrm{C}$ with an increment of $0.2{ }^{\circ} \mathrm{C}$ for $5 \mathrm{~s}$. Threshold cycle values were calculated via regression analysis using CFX manager V 2.0 (BioRad). Differentially expressed genes were identified and analysed with the option 'gene study' of CFX manager software. The genes pvuA, dnaE, rec $\mathrm{A}$ and a locus of the $16 \mathrm{~S}-23 \mathrm{~S}$ intergenic spacer region (1623S) were used for normalization via $\Delta \Delta C(\mathrm{q})$-method.

\section{Availability of supporting data}

The transcriptomics data were supplied as experiment GSE60815 at Gene Expression Omnibus according to MIAME regulations. Available at: http://www.ncbi.nlm.nih.gov/geo/query/acc.cgi?acc=GSE60815.

\section{Additional files}

Additional file 1: DAVID categories. Additional file includes DAVID data for all temperatures. (XLSX $25 \mathrm{~kb}$ )

Additional file 2: Differentially expressed genes with $\log _{2}$ fold change $>1.5$ and adjusted $p$-value $<0.05$ in each condition.

Additional file includes the homolog and antagonistic reacting genes. (XLSX $192 \mathrm{~kb}$ )

\section{Abbreviations}

DAVID: Database for Annotation, Visualization and Integrated Discovery; T3SS-1: Type three secretion system one; T6SS: Type six secretion system; $\mathrm{TDH}$ : Thermostable direct hemolysin; TRH: Thermostable direct hemolysin related hemolysin.

\section{Competing interests}

The authors declare that they have no interests in competition.

Authors' contributions

SU conducted and performed the RT-PCR, GRT-PCR and microarray experiments. TA and STH participated in study design, data analysis, manuscript drafting and editing. AT, SAH and RA assisted with data analysis and manuscript revisions. All authors read and approved the manuscript. 


\section{Acknowledgements}

We acknowledge Kathrin Oeleker for assistance in performing strain cultivations. The project was funded by the German Ministry of Education and Research (BMBF) within the VibrioNet project.

\section{Author details \\ ${ }^{1}$ Institute of Food Hygiene, Freie Universität Berlin, Berlin, Germany. ${ }^{2}$ Department of Chemistry and Bioengineering, Tampere University of Technology, Tampere, Finland. ${ }^{3}$ Department of Signal Processing, Tampere University of Technology, Tampere, Finland. ${ }^{4}$ School of Health Sciences, University of Tampere, Tampere, Finland.}

\section{Received: 20 February 2015 Accepted: 12 October 2015}

\section{Published online: 23 October 2015}

\section{References}

1. Potasman I, Paz A, Odeh M. Infectious outbreaks associated with bivalve shellfish consumption: a worldwide perspective. Clin Infect Dis. 2002;35:921-8.

2. Gopal S, Otta SK, Kumar S, Karunasagar I, Nishibuchi M. The occurrence of Vibrio species in tropical shrimp culture environments; implications for food safety. Int J Food Microbiol. 2005;102:151-9.

3. Slayton RB, Newton AE, Depaola A, Jones JL, Mahon BE. Clam-associated vibriosis, USA. Epidemiol Infect. 1988-2010;2013:1-6.

4. Vieira RH, de Sousa OV, Costa RA, Theophilo GN, Macrae A, et al. Raw oysters can be a risk for infections. Braz J Infect Dis. 2010;14:66-70.

5. Su YC, Liu C. Vibrio parahaemolyticus: a concern of seafood safety. Food Microbiol. 2007;24:549-58.

6. McLaughlin JB, DePaola A, Bopp CA, Martinek KA, Napolilli NP, et al. Outbreak of Vibrio parahaemolyticus gastroenteritis associated with Alaskan oysters. N Engl J Med. 2005;353:1463-70.

7. Chiang ML, Ho WL, Chou CC. Response of Vibrio parahaemolyticus to ethanol shock. Food Microbiol. 2006;23:461-7.

8. Chang CM, Chiang ML, Chou CC. Response of heat-shocked Vibrio parahaemolyticus to subsequent physical and chemical stresses. J Food Prot. 2004;67:2183-8.

9. Chiang ML, Chou CC. Survival of Vibrio parahaemolyticus under environmental stresses as influenced by growth phase and pre-adaptation treatment. Food Microbiol. 2009;26:391-5.

10. Phadtare S, Alsina J, Inouye M. Cold-shock response and cold-shock proteins. Curr Opin Microbiol. 1999;2:175-80.

11. Weber MH, Marahiel MA. Bacterial cold shock responses. Sci Prog. 2003;86:9-75

12. Phadtare S. Recent developments in bacterial cold-shock response. Curr Issues Mol Biol. 2004;6:125-36.

13. Yang L, Zhou D, Liu X, Han H, Zhan L, et al. Cold-induced gene expression profiles of Vibrio parahaemolyticus: a time-course analysis. FEMS Microbiol Lett. 2009;291:50-8.

14. Gophna U, Ron EZ. Virulence and the heat shock response. Int J Med Microbiol. 2003;292:453-61.

15. Segal G, Ron EZ. Regulation of heat-shock response in bacteria. Ann N Y Acad Sci. 1998:851:147-51.

16. Yura $T$, Nagai $H$, Mori $H$. Regulation of the heat-shock response in bacteria. Annu Rev Microbiol. 1993:47:321-50.

17. Bukau B. Regulation of the Escherichia coli heat-shock response. Mol Microbiol. 1993:9:671-80

18. Schroder H, Langer T, Hartl FU, Bukau B. DnaK, DnaJ and GrpE form a cellular chaperone machinery capable of repairing heat-induced protein damage. EMBO J. 1993;12:4137-44.

19. Wong HC, Peng PY, Lan SL, Chen YC, Lu KH, et al. Effects of heat shock on the thermotolerance, protein composition, and toxin production of Vibrio parahaemolyticus. J Food Prot. 2002;65:499-507.

20. Chiang ML, Chou CC. Expression of superoxide dismutase, catalase and thermostable direct hemolysin by, and growth in the presence of various nitrogen and carbon sources of heat-shocked and ethanol-shocked Vibrio parahaemolyticus. Int J Food Microbiol. 2008;121:268-74.

21. Mahoney JC, Gerding MJ, Jones SH, Whistler CA. Comparison of the pathogenic potentials of environmental and clinical Vibrio parahaemolyticus strains indicates a role for temperature regulation in virulence. Appl Environ Microbiol. 2010;76:7459-65.

22. Maurelli AT, Blackmon B, Curtiss R. 3rd Temperature-dependent expression of virulence genes in Shigella species. Infect Immun. 1984;43:195-201.
23. Straley SC, Perry RD. Environmental modulation of gene expression and pathogenesis in Yersinia. Trends Microbiol. 1995;3:310-7.

24. Rappuoli R, Arico B, Scarlato V. Thermoregulation and reversible differentiation in Bordetella: a model for pathogenic bacteria. Mol Microbiol. 1992;6:2209-2211.35.

25. Gonzalez-Escalona N, Martinez-Urtaza J, Romero J, Espejo RT, Jaykus LA, et al. Determination of molecular phylogenetics of Vibrio parahaemolyticus strains by multilocus sequence typing. J Bacteriol. 2008;190:2831-40.

26. Zhu J, Shimizu K. The effect of pfl gene knockout on the metabolism for optically pure D-lactate production by Escherichia coli. Appl Microbiol Biotechnol. 2004;64(3):367-75.

27. Fields PA. Review: Protein function at thermal extremes: balancing stability and flexibility. Comp Biochem Physiol A Mol Integr Physiol. 2001;129(2-3):417-31.

28. Badger $\mathrm{J}$, Miller VL. Role of RpoS in survival of Yersinia enterocolitica to a variety of environmental stresses. J Bacteriol. 1995;177:5370-3.

29. Persson $B C$, Jäger $G$, Gustafsson $C$. The spoU gene of Escherichia coli, the fourth gene of the spoT operon, is essential for tRNA (Gm18) 2'-O-methyltransferase activity. Nucleic Acids Res. 1997:25(20):4093-7.

30. Wong SM, Akerley BJ. Environmental and genetic regulation of the phosphorylcholine epitope of Haemophilus influenzae lipooligosaccharide. Mol Microbiol. 2005;55(3):724-38.

31. Vorachek-Warren MK, Carty SM, Lin S, Cotter RJ, et al. An Escherichia coli mutant lacking the cold shock-induced palmitoleoyltransferase of lipid A biosynthesis: absence of unsaturated acyl chains and antibiotic hypersensitivity at 12 degrees C. J Biol Chem. 2002;277(16):14186-93.

32. Makino K, Oshima K, Kurokawa K, Yokoyama K, Uda T, et al. Genome sequence of Vibrio parahaemolyticus: a pathogenic mechanism distinct from that of $V$. cholerae. Lancet. 2003;361:743-9.

33. Ma L, Zhang $Y$, Yan $X$, Guo L, Wang $L$, et al. Expression of the type $V$ secretion system 1 component Hcp1 is indirectly repressed by OpaR in Vibrio parahaemolyticus. ScientificWorldJournal. 2012;982140.

34. Somerville GA, Proctor RA. At the crossroads of bacterial metabolism and virulence factor synthesis in Staphylococci. Microbiol Mol Biol Rev. 2009:73:233-48.

35. Salomon D, Gonzalez H, Updegraff BL, Orth K. Vibrio parahaemolyticus Type VI Secretion System 1 is activated in marine conditions to target bacteria, and is differentially regulated from System 2. PLoS One. 2013;8, e61086.

36. Nasu H, lida T, Sugahara T, Yamaichi Y, Park KS, et al. A filamentous phage associated with recent pandemic Vibrio parahaemolyticus O3:K6 strains. J Clin Microbiol. 2000:38:2156-61.

37. Thompson FL, Cleenwerck I, Swings J, Matsuyama J, lida T. Genomic diversity and homologous recombination in Vibrio parahaemolyticus as revealed by amplified fragment length polymorphism (AFLP) and multilocus sequence analysis (MLSA). Microbes Environ. 2007;22:373-9.

38. Bolstad BM, Irizarry RA, Astrand M, Speed TP. A comparison of normalization methods for high density oligonucleotide array data based on variance and bias. Bioinformatics. 2003:19:185-93.

39. Smyth GK. Linear models and empirical Bayes methods for assessing differential expression in microarray experiments. Stat Appl Genet Mol Biol. 2004;3(1):Art 3

40. Benjamini $Y$, Yekutieli $D$. The control of the false discovery rate in multiple testing under dependency. Ann Stat. 2001;4:1165-88.

41. da Huang W, Sherman BT, Lempicki RA. Systematic and integrative analysis of large gene lists using DAVID bioinformatics resources. Nat Protoc. 2009;4:44-57.

42. da Huang W, Sherman BT, Lempicki RA. Bioinformatics enrichment tools: paths toward the comprehensive functional analysis of large gene lists. Nucleic Acids Res. 2009:37:1-13.

43. Sturn A, Quackenbush J, Trajanoski Z. Genesis: cluster analysis of microarray data. Bioinformatics. 2002;18:207-8.

44. Krzywinski Ml, Schein JE, Birol I, Connors J, Gascoyne R, et al. Circos: An information aesthetic for comparative genomics. Genome Res. 2009;19(9):1639-45.

45. Kong RY, Lee SK, Law TW, Law SH, Wu RS. Rapid detection of six types of bacterial pathogens in marine waters by multiplex PCR. Water Res. 2002:36:2802-12. 